This document is the accepted manuscript version of a published work that appeared in final form in Journal of physical chemistry letters, copyright $\odot$ American Chemical Society after peer review and technical editing by the publisher. To access the final edited and published work see

DOI 10.1021/jz5026118

The posting must be for non-commercial purposes and not violate the ACS' "Ethical Guide.

This version is published under a "All rights reserved" license. 


\title{
Assembly of Ferrocene Molecules on Metal Surfaces Revisited
}

\author{
Maider Ormaza, ${ }^{*, \dagger}$ Paula Abufager, ${ }^{\ddagger}, N^{-}$Nicolas Bachellier, ${ }^{\dagger}$ Roberto Robles, ${ }^{\ddagger}, \S$ \\ Martin Verot,|| Tangui Le Bahers," Marie-Laure Bocquet, || Nicolas Lorente, ${ }^{\ddagger}, \S$ \\ and Laurent Limot ${ }^{\dagger}$
}

Institut de Physique et Chimie des Matériaux de Strasbourg, Université de Strasbourg, UMR CNRS 7504, 67034 Strasbourg, France, ICN2 - Institut Català de Nanociència $i$ Nanotecnologia, Campus UAB, 08193 Bellaterra (Barcelona), Spain, Instituto de Física de Rosario, Consejo Nacional de Investigaciones Científicas y Técnicas (CONICET) and Universidad Nacional de Rosario, Av. Pellegrini 250 (2000) Rosario, Argentina, CSIC -

Consejo Superior de Investigaciones Cientificas, ICN2 Building, Campus UAB, 08193 Bellaterra (Barcelona), Spain, and Université de Lyon, Université Claude Bernard Lyon 1, Centre National de Recherche Scientifique, ENS Lyon, 46 allèe d'Italie, 69007 Lyon Cedex 0\%, France

E-mail: maider.ormaza@ipcms.unistra.fr

\footnotetext{
${ }^{*}$ To whom correspondence should be addressed

†Institut de Physique et Chimie des Matériaux de Strasbourg, Université de Strasbourg, UMR CNRS 7504, 67034 Strasbourg, France

${ }^{\ddagger}$ ICN2 - Institut Català de Nanociència i Nanotecnologia, Campus UAB, 08193 Bellaterra (Barcelona), Spain

"Instituto de Física de Rosario, Consejo Nacional de Investigaciones Científicas y Técnicas (CONICET) and Universidad Nacional de Rosario, Av. Pellegrini 250 (2000) Rosario, Argentina

${ }^{\S}$ CSIC - Consejo Superior de Investigaciones Cientificas, ICN2 Building, Campus UAB, 08193 Bellaterra (Barcelona), Spain

"Université de Lyon, Université Claude Bernard Lyon 1, Centre National de Recherche Scientifique, ENS Lyon, 46 allèe d'Italie, 69007 Lyon Cedex 07, France
} 


\begin{abstract}
Metallocene $\left(\mathrm{MCp}_{2}\right)$ wires have recently attracted considerable interest in relation to molecular spintronics due to predictions concerning their half-metallic nature. This exciting prospect is however hampered by the little and often-contradictory knowledge we have concerning the metallocene self-assembly and interaction with a metal. Here, we elucidate these aspects by focusing on the adsorption of ferrocene on $\mathrm{Cu}(111)$ and $\mathrm{Cu}(100)$. Combining low-temperature scanning tunneling microscopy and density functional theory calculations, we demonstrate that the two-dimensional molecular arrangement consists of vertical- and horizontal-lying molecules. The non-covalent Tshape interactions between $\mathrm{Cp}$ rings of vertical and horizontal molecules are essential for the stability of the physisorbed molecular layer. These results provide a fresh insight into ferrocene adsorption on surfaces, and may serve as an archetypal reference for future work with this important variety of organometallic molecules.
\end{abstract}

\title{
Graphical TOC Entry
}

\section{Keywords}

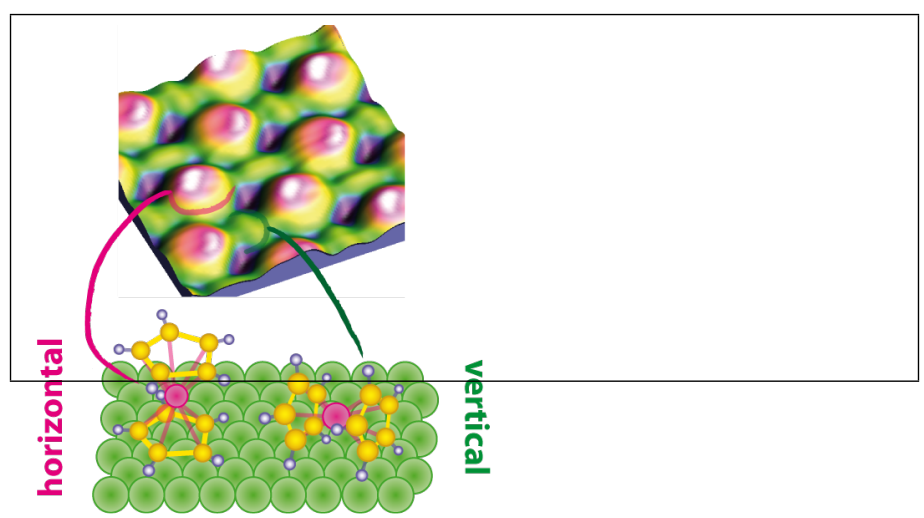

Ferrocene, adsorption, self-assembly, $\mathrm{Cu}(111), \mathrm{Cu}(100)$, scanning tunneling microscopy, density functional theory 
Metallocenes were discovered in the fifties and have boosted the development of organometallic chemistry, earning, in part, to Fischer and Wilkinson the Nobel Prize in chemistry in 1973. They are organometallic sandwich compounds of simplified architecture as build on two cyclopentadienyl rings $\left(\mathrm{C}_{5} \mathrm{H}_{5}^{-}, \mathrm{Cp}\right)$ bound through a metal center, for example by $\mathrm{Fe}^{2+}$ in the case of ferrocene (see Figure 1). In the past decade, metallocenes were again in the spotlight in relation to molecular spintronics. This emerging technology exploits the spin to convey information in hybrid metal-molecule devices. ${ }^{1}$ Molecules are extremely appealing, as they ensure high-device efficiency and offer the unique possibility of having build-in spin functionalities. Numerous theoretical investigations have demonstrated that metallocene wires can play a prominent role in molecular spintronics as they can produce a nearly ideal spin-polarized current, in other words, a current with $100 \%$ spin polarization. ${ }^{2-8}$ Despite this exciting prospect, the design of devices incorporating metallocenes is hold up by the limited knowledge we have concerning their interaction with a metal. After all, the device performance will be dictated by the adsorption geometry, molecular self-assembly and spin-state at a metal surface. ${ }^{9}$

There is little consensus on the way these molecules adsorb onto surfaces. Several studies have reported an associative adsorption at low temperature of ferrocene $\left(\mathrm{FeCp}_{2}\right)$ on $\mathrm{Ag},{ }^{10-12}$ $\mathrm{Cu},{ }^{11}$ graphite ${ }^{13,14}$ and $\mathrm{Mo}^{15}$ by means of photoemission (PES) and electron energy loss spectroscopy (EELS), claiming a preferential orientation of the molecular axis perpendicular or parallel to the surface depending on the substrate (Figure 1a). On the other hand, based on STM measurements, K. F. Braun et al. ${ }^{16}$ proposed a dissociative adsorption model of $\mathrm{FeCp}_{2}$ on $\mathrm{Au}(111)$, while an associative adsorption has been addressed for various ferrocenederived molecules on the same substrate. ${ }^{17,18}$ B. W. Heinrich and coworkers ${ }^{19}$ presented a molecularly physisorbed ferrocene on $\mathrm{Cu}(111)$ lying with the molecular axis perpendicular to the surface.

The adsorption of metallocenes remains elusive up to now. In this work we aim to clarify the self-assembly, adsorption geometry and interactions of ferrocene molecules deposited on a 


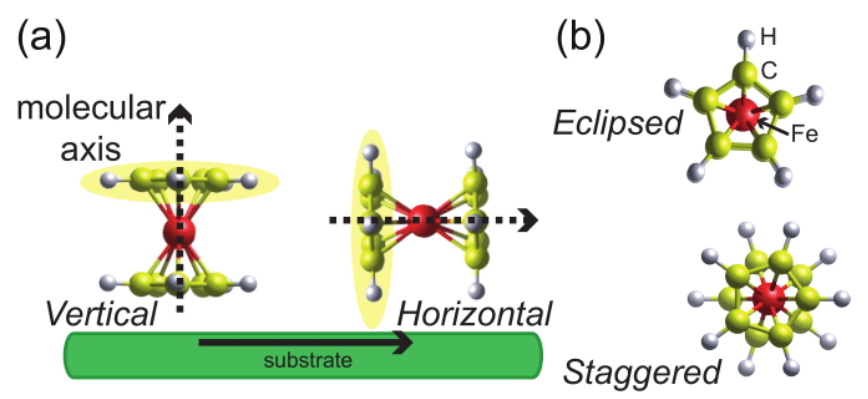

Figure 1: (a) Different positions, vertical and horizontal, of a ferrocene with respect to the surface. The molecular axis in both cases is indicated with a dashed arrow. White, yellow, and red balls represent H, C, and Fe atoms, respectively. (b) Eclipsed and staggered conformations of a ferrocene molecule.

metallic surface. By means of scanning tunneling microscopy (STM) and density functional theory (DFT), we show how associatively adsorbed $\mathrm{FeCp}_{2}$ molecules self-assemble on a $\mathrm{Cu}$ surface adopting a configuration which is independent of the surface orientation, dismissing the idea of a unique preferential orientation of the molecular axis with respect to the substrate suggested by previous works. In fact, the stability of the two-dimensional structure depends crucially on a combination of vertical (molecular axis perpendicular to the surface, Figure 1a) and horizontal (axis parallel to the surface) molecules having their Cp rings in a so-called eclipsed configuration ( $\mathrm{D}_{5 h}$ symmetry, Figure $\left.1 \mathrm{~b}\right)$. Interestingly, this arrangement bears similarities with both the gas phase and the bulk structure of ferrocene. Similar to the ferrocene crystal (see Figure 4e), the presence of the two molecules, vertical and horizontal, ensures the cohesion of the crystal through T-shape interactions, but as in the gas phase-and unlike bulk ferrocene - the eclipsed configuration is favored over the staggered configuration ( $\mathrm{D}_{5 d}$ symmetry, Figure 1b).

The adsorption of $\mathrm{FeCp}_{2}$ on a cold $\mathrm{Cu}(111)$ surface gives rise to long-range well-ordered molecular layers with almost no defects and exhibiting an apparent height of $3.1 \AA$ (Figure 2a). Two different molecular configurations, which we label "compact" and "zigzag" (see Figure $2 \mathrm{~b}$ and $2 \mathrm{~d}$ respectively), appear equally distributed across the surface. Identical results are observed for $\mathrm{FeCp}_{2}$ deposited on $\mathrm{Cu}(100)$ (see supporting information). 

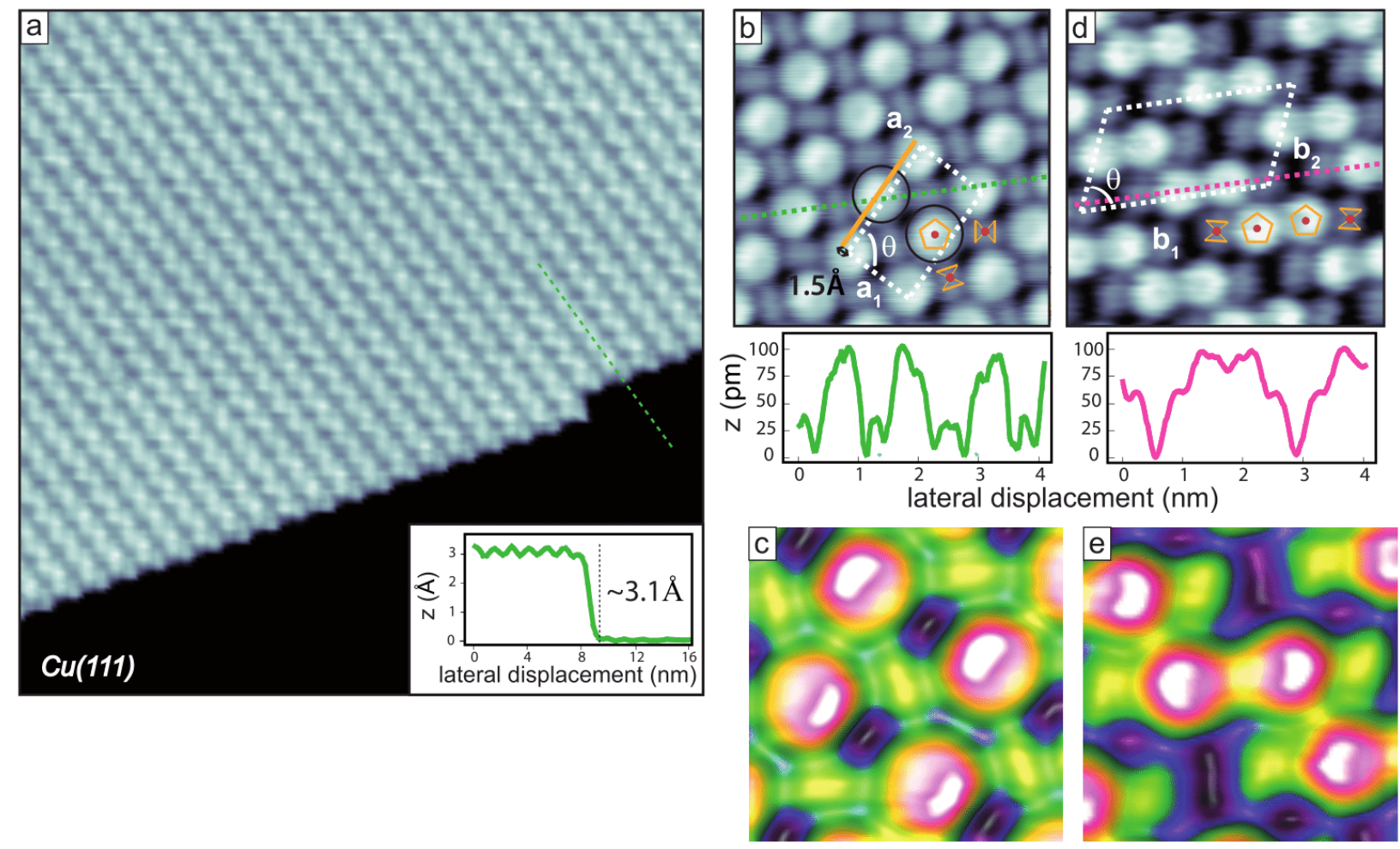

Figure 2: (a) STM image of 0.6 monolayer $\mathrm{FeCp}_{2}$ deposited on $\mathrm{Cu}(111)(+1 \mathrm{~V}, 0.5 \mathrm{nA})$. Inset: Height profile of the molecular monolayer. Two different configurations are observed on $\mathrm{Cu}(111)$ : (b) compact $(-1 \mathrm{~V}, 0.5 \mathrm{nA})$ and (d) zigzag $(+0.1 \mathrm{~V}, 0.2 \mathrm{nA}$ ). The unit cells (white dashed lines), composed of vertical and horizontal molecules, as well as the line profiles of both arrangements are shown in each image. (b-d) Close up view of the two configurations, highlighting the asymmetry of the rings in the vertical molecules. Image sizes: (a) $30 \times 30 \mathrm{~nm}^{2}$, (b-d) $4 \times 4 \mathrm{~nm}^{2}$, (c-e) $2 \times 2 \mathrm{~nm}^{2}$.

In the compact configuration, elongated rod-like protrusions are observed in between ring-like protrusions. We assign these ring-like units to the $\mathrm{Cp}$ rings of vertical $\mathrm{FeCp}_{2}$, while the rod-like protrusions around them, showing a height $0.5 \AA$ smaller and therefore appearing dimmer in the images, are assigned to horizontal $\mathrm{FeCp}_{2}$. The corrugation observed along the dashed green line in Figure $2 \mathrm{~b}$ due to the presence of both, vertical and horizontal, molecules is shown in the line profile below the image. The rectangular unit cell of the compact configuration is marked in white. The cell, with lattice parameters $\mathrm{a}_{1}, \mathrm{a}_{2}$ and angle $\theta$ (see Table 1 for values), contains four molecules, two horizontal and two vertical. As shown, consecutive vertical molecules in the $\mathrm{a}_{2}$ direction do not lie exactly in the same axis 
(see the orange axis and the molecules marked with circles in Figure 2b), they are shifted by $1.5 \AA$. Moreover, vertical molecules present an asymmetry in the $\mathrm{a}_{1}$ direction, being brighter on one side of the ring; vertical molecules in the center and in the corner of the unit cell show the asymmetry at different sides of the ring. This asymmetry can be clearly observed in Figure 2c, and, as we show later, indicates that the molecules are tilted.

Figure $2 \mathrm{~d}$ presents the zigzag configuration in which two ring-like protrusions, corresponding to vertical molecules, form a dimer-like pair. They are flanked by horizontal molecules which have a dimmer contrast in the image. The line profile taken along the pink dashed line shows the corrugation of the molecular assembly. Similarly to the compact case, the apparent height difference between vertical and horizontal molecules is around $0.5 \AA$. The distance between the two adjacent dimer-like vertical molecules is $(6.2 \pm 0.2) \AA$, which is small compared to the $(8.0 \pm 0.2) \AA$ found for the compact arrangement. The unit cell is a parallelogram (see parameters in Table 1), as shown in white in Figure 2d. A similar ferrocene pairing has been also observed for ferrocene-derived molecules (FcCOOH and Fc- $\left.\left(\mathrm{CH}_{2}\right)_{n}-\mathrm{Fc}\right)$ deposited on metallic surfaces. ${ }^{18,20}$

Although the identification of vertical ferrocene molecules is straightforward, determining the presence of the horizontal molecules is experimentally challenging. Therefore, to confirm the proposed adsorption model for ferrocene we rotated some molecules from a vertical (horizontal) to a horizontal (vertical) position by using the STM tip ${ }^{21}$ as shown in the before-and-after images in Figure 3. The molecular structure of $\mathrm{FeCp}_{2}$ is superimposed in the images below (the structure used has a random orientation). Upon application of a bias pulse of $2 \mathrm{~V}$, molecules rotate out-of-plane from horizontal to vertical (molecules noted 1 and 2), or viceversa (molecules noted 3 and 4). There are likely in-plane rotations more difficult to identify. Clearly, when the vertical axis of $\mathrm{FeCp}_{2}$ is rotated nearly $90^{\circ}$ parallel to the surface, the ring-like feature is lost, confirming the presence of both vertical and horizontal molecules on the surface. 

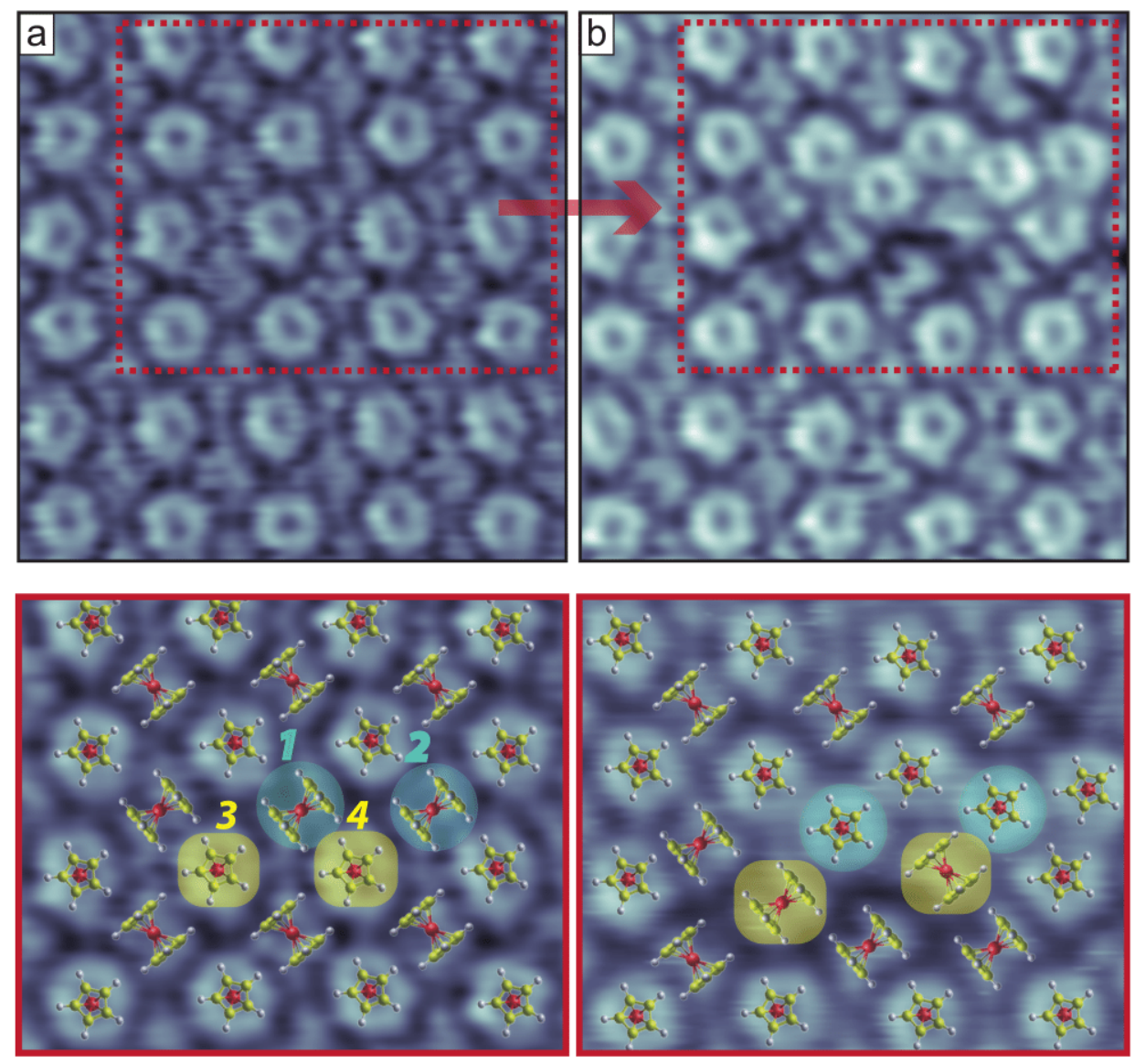

Figure 3: STM images $(+1 \mathrm{~V}, 1 \mathrm{nA})$ of the compact configuration on $\mathrm{Cu}(111)$ before and after some molecules turned from a vertical to a horizontal position, or vice versa, via tipassisted manipulation. The images below correspond to the marked squares in the pictures above, with the molecules superimposed to highlight the molecules that have rotated (1-4). Notice that a molecular tip has been employed in order to enhance the observed features. Additionally, a laplacian filter has been applied. Image sizes: $(\mathrm{a}-\mathrm{b}) 4.5 \times 4.5 \mathrm{~nm}^{2}$.

In order to study the stability and electronic properties of the structures proposed for the molecular layer on $\mathrm{Cu}(111)$ we have performed extensive DFT calculations and simulated the corresponding STM images. First, we started by studying the intra-molecular structure of ferrocene molecules. As shown in Figure 1b, two different configurations can be found for a ferrocene molecule: eclipsed and staggered. The former one corresponds to the most stable structure in the gas phase ${ }^{22-24}$ while the latter is the most common one in the solid state. ${ }^{25}$ We found that the eclipsed configuration is more stable by $45 \mathrm{meV}$ on the surface. According to our calculations, no matter what initial configuration is chosen, the eclipsed 

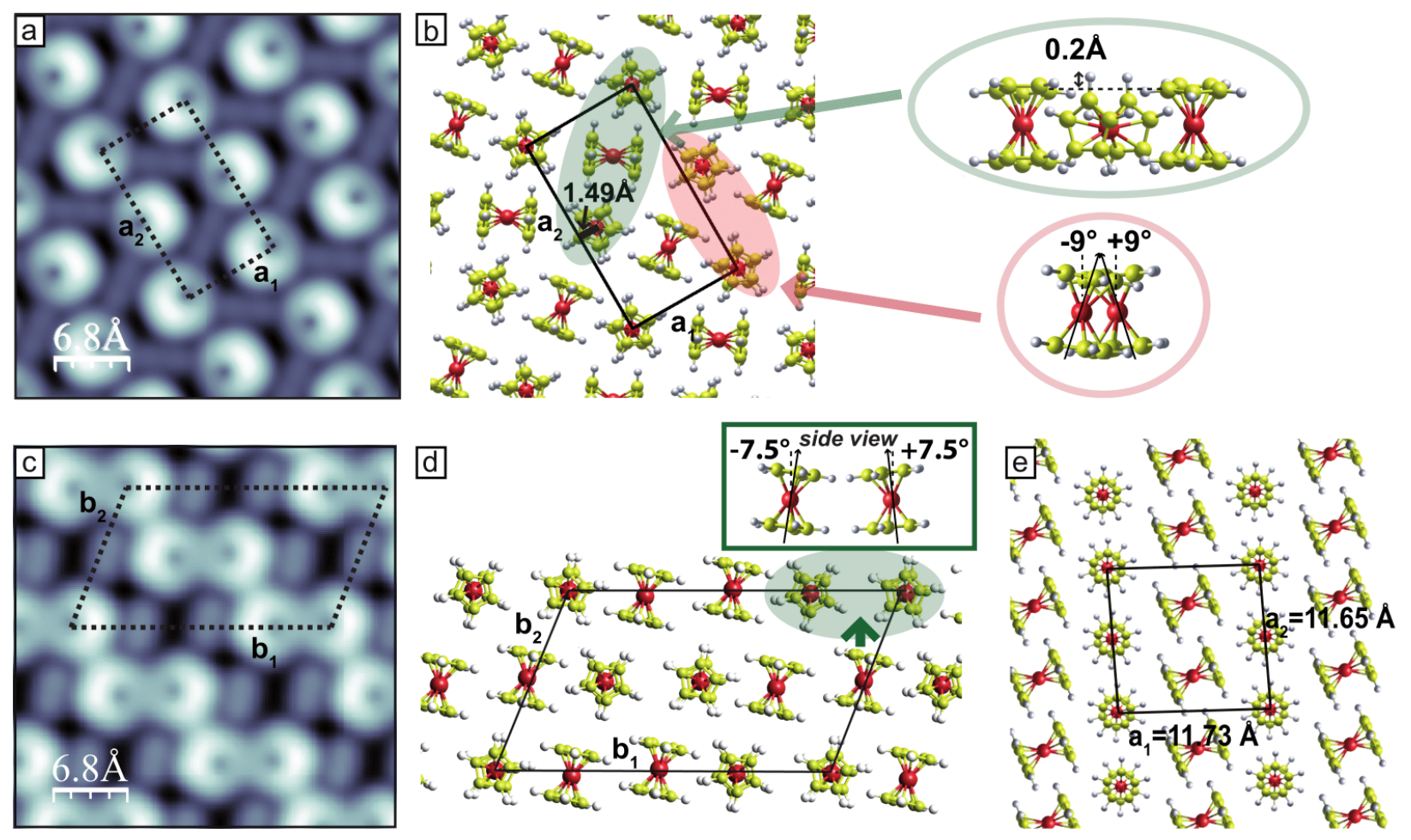

Figure 4: (a-c) Simulated STM topographic images for a bias of $1 \mathrm{~V}$ corresponding to the relaxed (b) compact and d) zigzag structure on the $\mathrm{Cu}(111)$ surface. Lateral views of the indicated molecules are shown in both cases, in order to present the relative position and tilt between molecules. (e) Crystallographic (100) plane of the bulk solid ferrocene.

structure is always preferred for both horizontal and vertical molecules. In the molecular layer discussed hereafter we find exclusively molecules in the eclipsed configuration.

We focused then on the inter-molecular arrangement of both compact and zigzag configurations. Let us first concentrate on the analysis of the compact configuration. In Figures $4 \mathrm{a}$ and $4 \mathrm{~b}$, different views of the most stable calculated structure, together with a simulated STM image are shown. The rectangular unit cell observed in the STM images (Figure 2b) corresponds within experimental accuracy to a $2 \times 6 \sqrt{3}$ unit cell in the $\mathrm{Cu}(111)$ surface (see Table 1). The stability of the proposed structure has been tested using this unit cell.

Comparing the simulated STM image (Figure 4a) with the experimental one (Figure $2 \mathrm{~b})$, we observe that the main features of the image are reproduced. The structure shown in Figure $4 \mathrm{~b}$, in which the minimum distance from the surface to the molecule is $2.3 \AA$, 
Table 1: Comparison of the unit cell parameters $\left(a_{1}, a_{2}, b_{1}, b_{2}, \theta\right)$ and of the energetics of the adsorption for both compact and zigzag arrangements. $E_{a d s}$ (adsorption energy per molecule), $E_{a d s}^{v d W}$ (vdW contribution per molecule coming from the molecule-substrate interaction), $E_{\text {coh }}^{\text {free }}$ (cohesive energy per molecule for the free-standing overlayer), $q$ (molecule-substrate charge tranfer per unit cell), $d_{\text {mol-surf }}$ (molecule-surface distance).

\begin{tabular}{|c|c|c|c|c|c|}
\hline & & & $\mathbf{a}_{1}(\AA)$ & $\mathrm{a}_{2}(\AA)$ & $\theta\left(^{\circ}\right)$ \\
\hline \multirow[t]{4}{*}{ Compact } & Unit Cell & Experiment & $8.9 \pm 0.3$ & $15.5 \pm 0.3$ & $90 \pm 3$ \\
\hline & \multirow{3}{*}{$\begin{array}{c}\mathbf{E}_{a d s}(\mathbf{e V}) \\
1.35\end{array}$} & Theory $(2 \sqrt{3} \times 6)$ & 8.75 & 15.15 & 90 \\
\hline & & $\mathbf{E}_{a d s}^{v d W}(\mathbf{e V})$ & $\mathbf{E}_{c o h}^{\text {free }}(\mathbf{e V})$ & $\mathbf{q}\left(e^{-}\right)$ & $\mathbf{d}_{\text {mol-surf }}(\AA)$ \\
\hline & & 1.05 & 0.48 & 0.11 & 2.3 \\
\hline & & & $\mathbf{b}_{1}(\AA)$ & $\mathbf{b}_{2}(\AA)$ & $\theta\left(^{\circ}\right)$ \\
\hline \multirow[t]{4}{*}{ Zigzag } & Unit Cell & Experiment & $23.0 \pm 0.4$ & $13.0 \pm 0.4$ & $71 \pm 3$ \\
\hline & & Theory & 23.15 & 13.37 & 68 \\
\hline & $\mathbf{E}_{a d s}(\mathbf{e V})$ & $\mathbf{E}_{a d s}^{v d W}(\mathbf{e V})$ & $\mathbf{E}_{c o h}^{\text {free }}(\mathbf{e V})$ & $\mathbf{q}\left(e^{-}\right)$ & $\mathbf{d}_{\text {mol-surf }}(\AA)$ \\
\hline & 1.26 & 1.02 & 0.43 & 0.18 & 2.4 \\
\hline
\end{tabular}

allows us to explain all these signatures. Rod-like and ring-like protrusions in the image correspond to vertical and horizontal molecules respectively. A strong contrast difference is observed between both molecules in the STM images. At first glance, this contrast appears counterintuitive as horizontal molecules appear dimmer, despite some of their $\mathrm{H}$ atoms are $0.2 \AA$ further away into vacuum compared to the $\mathrm{Cp}$ ring of the vertical molecules (side view of Figure $4 \mathrm{~b})$. This apparent contradiction can be easily lifted by recalling that the tunneling transmission probability between the STM tip and the molecule depends on the tip and molecular orbitals. Orbitals that extend into the vacuum, such as $s, p_{z}$ or $d_{z}^{2}$, will be favored in the tunneling process. In the present case, for vertical molecules, the $d$-manifold orbital of Fe lies around the Fermi level and hybridizes with the $p$ orbitals perpendicular to the $\mathrm{Cp}$ rings that protude into the vacuum, enhancing the coupling with the tip orbitals. In contrast, for horizontal molecules, the $p$ orbitals of the organic ring lie in a plane parallel to the surface and do not point towards the STM tip. Hence, the simulated apparent height of the vertical molecules is $0.5 \AA$ higher, in agreement with the experimentally measured line 
profiles shown in Figure 2b.

Our calculations also reproduce subtle effects revealed by STM images. We observe a lateral displacement of $1.49 \AA$ between consecutive vertical molecules in the $a_{2}$ direction (marked in Figure 4b), measured as the distance between Fe atoms, which is consistent with the experimentally found shift of $1.5 \AA$ (marked in Figure 2b). Most importantly, we find that the slight asymmetry observed for the ring of the vertical molecules (Figure 2c) results from a tilt between successive vertical molecules in the $\mathrm{a}_{2}$ direction. This is a robust feature of the molecular layer as in our computation an initial configuration with no tilt always relaxes until a tilt is present. The origin of the tilt lies in the presence of the horizontal molecules. In particular, we found that the most stable situation is the one in which the horizontal molecules have two $\mathrm{H}$ atoms of each $\mathrm{Cp}$ ring pointing towards the surface, in this case this leaves a single $\mathrm{H}$ atom per $\mathrm{Cp}$ ring pointing into the vacuum. This configuration gives rise to a $18^{\circ}$ tilt between vertical molecules, as shown in the lateral view in Figure $4 \mathrm{~b}$.

In order to understand the intermolecular interactions, the nature of the interaction between two type of $\mathrm{FeCp}_{2}$ molecules was investigated. We found that the adsorption energy per molecule is $1.35 \mathrm{eV}$, where the contribution from the van der Waals (vdW) dispersion forces amounts to $1.05 \mathrm{eV}$ (see theoretical methods). Comparing the difference between both numbers $(0.30 \mathrm{eV})$ to the cohesion energy of the free-standing monolayer $(0.48 \mathrm{eV})$, it can be concluded that the interaction between the molecules and the surface is due to vdW forces. The modest charge transfer per unit cell from the substrate to the molecules $\left(0.11 \mathrm{e}^{-}\right)$points to molecules being physisorbed on the surface. This conclusion is supported by the fact that the presence of the surface is not necessary to stabilize the molecular layer. Calculations of the unsupported monolayer show that the free-standing structure is stable, and it presents the main features of the supported situation, including the tilt of the vertical molecules. Moreover, when the layer is moved to different positions with respect to the copper surface (top, bridge, $h c p, f c c$ ) the energy cost is only of the order of $0.1 \mathrm{eV}$ per unit cell. Additionally, experimental results indicating that the same molecular assembly is observed independently 
of the copper surface symmetry reinforce the idea of molecular physisorption (see supporting material).

As shown in Figure 4e, the (100) plane of pure ferrocene bulk (monoclinic phase P21/a) exhibits a very similar arrangement to the compact structure observed on the surface. This confirms that the alternative orientation of a ferrocene network is frequently encountered even outside surface science. However, it can be noticed that in the primitive cell observed and computed for the compact structure (Figure 4b), adjacent eclipsed horizontal molecules are oriented differently, contrary to the unit cell of staggered ferrocenes shown by the bulk in Figure 4e. As in the crystalline structure, in this case we have also seen how the cohesion of the layer mainly comes from vdW interactions.

To conclude, we consider now the zigzag structure. Due to the size of the parallelogram unit cell (see parameters in Table 1), we have just performed a few calculations to confirm the stability of the proposed structure. Figure 4c shows the simulated topographic STM image corresponding to the most stable structure that has been found (Figure 4d), which reproduces with high accuracy the main features of the experimental image (Figure 2d). This structure shares some common features with the compact structure. First, the same reversal of the apparent height is observed, in which horizontal molecules always appear dimmer in the images, although the highest atom of horizontal molecules in this case is placed $0.4 \AA$ further from the surface than the topmost atom of the vertical ones. Second, we also find that four $\mathrm{H}$ atoms of the horizontal molecules have to point towards the surface in order to get a stable structure. Third, a $15^{\circ}$ tilt in two opposite directions is also observed for the vertical molecules (side view of Figure 4d), explaining the experimentally observed asymmetry between vertical molecules shown in Figure 2e.

According to an analysis of the energetics of the zigzag configuration, the adsorption energy per molecule is $1.26 \mathrm{eV}$, of which $1.02 \mathrm{eV}$ comes from the contribution of vdW forces. The interaction between the molecules and the copper surface is mainly related to vdW forces, as may be concluded after comparing these adsorption values with the cohesion energy of 
the free-standing monolayer $(0.43 \mathrm{eV})$. There is a moderate charge transfer per unit cell, $0.18 \mathrm{e}^{-}$, which is also indicating a weak molecule-substrate interaction. Table 1 summarizes the energetics of the adsorption of both, compact and zizag, configurations, showing that they are very similar, pointing in both cases to the same conclusion of a physisorbed layer.

We have presented a complete experimental and theoretical study of ferrocene molecules adsorbed onto copper surfaces, which solves the long-standing dilemma concerning the selfassembly of ferrocene on a metal surface. We demonstrate that ferrocene molecules adsorb associatively forming two different self-assemblies, compact and zigzag, which appear equally distributed on the surface. These arrangements, which include the presence of vertical and horizontal molecules, are lead by intermolecular interactions, in particular T-shape interactions, which are essential to stabilize the structures and to explain subtle submolecular features resolved in the STM images. As in the gas-phase ferrocene, the eclipsed configuration is found to be the most stable one for molecules adsorbed on a copper surface, while the presence of vertical and horizontal molecules on the surface is reminiscent of the crystallographic structure of bulk ferrocene. The fact that the same configurations, compact and zigzag, are found in differently oriented copper surfaces, together with the small charge transfer and the vdW interaction between the molecular monolayer and the $\mathrm{Cu}$ surface, confirms the physisorption of the molecules. We believe that these results may provide a solid guideline for future investigations on ferrocene, and eventually, related metallocenes on surfaces.

\section{Methods}

\section{Experiments}

Samples have been prepared in a ultrahigh vacuum (UHV) chamber at a base pressure of $10^{-10}$ mbar equipped with a LT-STM operating at $4.5 \mathrm{~K}$. The $\mathrm{Cu}(111)$ and $\mathrm{Cu}(100)$ metallic surfaces were cleaned by repeated $\mathrm{Ar}^{+}$ion bombardments and annealing treatments 
at $860 \mathrm{~K}$ until clean large terraces were obtained. $\mathrm{FeCp}_{2}$ molecules were sublimated at room-temperature in vacuo on the metallic cold surfaces $(\leq 100 \mathrm{~K})$ with a deposition rate of approximately 1.2 ML/min. After the molecular deposition, the samples were immediately cooled to $4.5 \mathrm{~K}$ for STM measurements. In situ cleaned W tips were used for measurements. WSxM software has been used for data analysis. ${ }^{26}$

\section{Theory}

We have studied the geometric and electronic structures of the adsorbed molecules by performing $a b$ initio density functional theory calculations as implemented in the VASP code. ${ }^{27}$ We have used a plane wave basis set to expand the wave functions with a cut-off energy of $400 \mathrm{eV}$. Core electrons have been treated using the projector augmented wave method, ${ }^{28,29}$ and we have used the generalized gradient approximation in the PBE form for the exchange and correlation functional. ${ }^{30}$ The long-range dispersion corrections have been treated using the DFT+D2 approach proposed by Grimme. ${ }^{31}$ Although this empirical vdW correction remains an approximation in light of the more recent self-consistent approaches, ${ }^{32}$ it readily corrects the major lack of GGA energies and enables a qualitative description of the interactions at play in the self-assembly process. Charge transfer has been obtained by performing a Bader analysis. ${ }^{33}$

The $\mathrm{Cu}(111)$ surface has been modeled using a slab geometry with five $\mathrm{Cu}$ layers and a vacuum region of $19 \AA$. We have kept fixed the bottom three $\mathrm{Cu}$ layers, allowing the two topmost layers and the ferrocene atoms to relax until forces were smaller than $0.02 \mathrm{eV} / \AA$. The D2 lattice constant of $\mathrm{Cu}$ has been used $(3.572 \AA)$. The compact structure has been simulated using a $6 \times 2 \sqrt{3}$ unit cell, with a $(3 \times 5 \times 1)$ Monkhorst-Pack mesh for the Brillouin zone integration. For the zigzag structure we have used a unit cell with $b_{1}=23.15 \AA, b_{2}=13.37 \AA$ lattice vectors. Due to the size of the system we have used the gamma point for the Brillouin zone integration. For the same reason, we also modelled the slab by only three layers of copper with the topmost layer relaxed. 
We have simulated STM topographic images of the structures within the Tersoff and Hamman theory ${ }^{34,35}$ using the method described by Bocquet et al. ${ }^{36}$

\section{Supporting Information Available}

Experimental study of the adsorption of $\mathrm{FeCp}_{2}$ on $\mathrm{Cu}(100)$. This material is available free of charge via the Internet at http://pubs.acs.org/.

\section{Acknowledgement}

This work has been supported by the Agence Nationale de la Recherche (Grant No. ANR13-BS10-0016). L.L. acknowledges also financial support from the Agence Nationale de la Recherche through projects LabEx NIE and LabEx CSC. R.R. and N.L. acknowledge financial support from Spanish MINECO (Grant No. MAT2012-38318- C03-02 with joint financing by FEDER Funds from the European Union). P.A. acknowledges financial support from CONICET. ICN2 acknowledges support from the Severo Ochoa Program (MINECO, Grant SEV-2013-0295).

\section{References}

(1) Xiong, Z. H.; Wu, D.; Vardeny, Z. V.; Shi, J. Giant Magnetoresistance in Organic Spin-Valves. Nature 2004, 427, 821-824.

(2) Zhou, L.; Yang, S.-W.; Ng, M.-F.; Sullivan, M. B.; Tan,; Shen, L. One-Dimensional IronCyclopentadienyl Sandwich Molecular Wire with Half Metallic, Negative Differential Resistance and High-Spin Filter Efficiency Properties. J. Am. Chem. Soc. 2008, 130, 4023-4027.

(3) Wang, L.; Cai, Z.; Wang, J.; Lu, J.; Luo, G.; Lai, L.; Zhou, J.; Qin, R.; Gao, Z.; Yu, D. et al. Novel One-Dimensional Organometallic Half Metals: Vanadium- 
Cyclopentadienyl, Vanadium-Cyclopentadienyl-Benzene, and Vanadium-Anthracene Wires. Nano Lett. 2008, 8, 3640-3644.

(4) Liu, R.; Ke, S.-H.; Baranger, H. U.; Yang, W. Organometallic Spintronics: A Dicobaltocene Switch. Nano Lett. 2005, 5, 1959-1962.

(5) García-Suárez, V. M.; Ferrer, J.; Lambert, C. J. Tuning the Electrical Conductivity of Nanotube-Encapsulated Metallocene Wires. Phys. Rev. Lett. 2006, 96, 106804.

(6) Shen, X.; Yi, Z.; Shen, Z.; Zhao, X.; Wu, J.; Hou, S.; Sanvito, S. The Spin Filter Effect of Iron-Cyclopentadienyl Multidecker Clusters: the Role of the Electrode Band Structure and the Coupling Strength. Nanotechnology 2009, 20, 385401.

(7) Yang, J.-F.; Zhou, L.; Han, Q.; Wang, X.-F. Bias-Controlled Giant Magnetoresistance through CyclopentadienylIron Multidecker Molecules. J. Phys. Chem. C 2012, 116, 19996-20001.

(8) Liu, R.; Ke, S.-H.; Yang, W.; Baranger, H. U. Cobaltocene as a Spin Filter. J. Chem. Phys. 2007, 127, 141104.

(9) Morari, C.; Rungger, I.; Rocha, A. R.; Sanvito, S.; Melinte, S.; Rignanese, G.-M. Electronic Transport Properties of 1,1-Ferrocene Dicarboxylic Acid Linked to Al(111) Electrodes. ACS Nano 2009, 3, 4137-4143.

(10) Welipitiya, D.; Green, A.; Woods, J. P.; Dowben, P. A.; Robertson, B. W.; Byun, D.; Zhang, J. Ultraviolet and Electron Radiation Induced Fragmentation of Adsorbed Ferrocene. J. Appl. Phys. 1996, 79, 8730-8734.

(11) Waldfried, C.; Welipitiya, D.; Hutchings, C. W.; de Silva, H. S. V.; Gallup, G. A.; Dowben, P. A.; Pai, W. W.; Zhang, J.; Wendelken, J. F.; Boag, N. M. Preferential Bonding Orientations of Ferrocene on Surfaces. J. Phys. Chem. B 1997, 101, 97829789. 
(12) Woodbridge, C. M.; Pugmire, D. L.; Johnson, R. C.; Boag, N. M.; Langell, M. A. HREELS and XPS Studies of Ferrocene on $\operatorname{Ag}(100)$. J. Phys. Chem. B 2000, 104, 3085-3093.

(13) Durston, P.; Palmer, R. Adsorption and Decomposition of Ferrocene on Graphite Studied by HREELS and STM. Surf. Sci. 1998, 400, 277-280.

(14) Svensson, K.; Bedson, T.; Palmer, R. Dissociation and Desorption of Ferrocene on Graphite by Low Energy Electron Impact. Surf. Sci. 2000, 451, 250-254.

(15) Dowben, P.; Waldfried, C.; Komesu, T.; Welipitiya, D.; McAvoy, T.; Vescovo, E. The Occupied and Unoccupied Electronic Structure of Adsorbed Ferrocene. Chem. Phys. Lett. 1998, 283, $44-50$.

(16) Braun, K.-F.; Iancu, V.; Pertaya, N.; Rieder, K.-H.; Hla, S.-W. Decompositional Incommensurate Growth of Ferrocene Molecules on a $\mathrm{Au}(111)$ Surface. Phys. Rev. Lett. 2006, 96, 246102.

(17) Quardokus, R. C.; Wasio, N. A.; Forrest, R. P.; Lent, C. S.; Corcelli, S. A.; Christie, J. A.; Henderson, K. W.; Alex Kandel, S. Adsorption of Diferrocenylacetylene on $\mathrm{Au}(111)$ Studied by Scanning Tunneling Microscopy. Phys. Chem. Chem. Phys. 2013, 15, 6973-6981.

(18) Wasio, N. A.; Quardokus, R. C.; Forrest, R. P.; Lent, C. S.; Corcelli, S. A.; Christie, J. A.; Henderson, K. W.; Alex Kandel, S. Self-Assembly of Hydrogen-Bonded Two-Dimensional Quasicrystals. Nature 2014, 507, 86-89.

(19) Heinrich, B. W.; Limot, L.; Rastei, M. V.; Iacovita, C.; Bucher, J. P.; Djimbi, D. M.; Massobrio, C.; Boero, M. Dispersion and Localization of Electronic States at a Ferrocene/Cu(111) Interface. Phys. Rev. Lett. 2011, 107, 216801. 
(20) Zhong, D.; Wedeking, K.; Blömker, T.; Erker, G.; Fuchs, H.; Chi, L. Multilevel Supramolecular Architectures Self-Assembled on Metal Surfaces. ACS Nano 2010, 4, $1997-2002$.

(21) Néel, N.; Limot, L.; Kröger, J.; Berndt, R. Rotation of $\mathrm{C}_{60}$ in a Single-Molecule Contact. Phys. Rev. B 2008, 77, 125431.

(22) Haaland, A.; Nilsson, J. E.; Olson, T.; Norin, T. The Determination of Barriers to Internal Rotation by Means of Electron Diffraction. Ferrocene and Ruthenocene. Acta Chem. Scand. 1968, 22, 2653-2670.

(23) Mohammadi, N.; Ganesan, A.; Chantler, C. T.; Wang, F. Differentiation of Ferrocene D5d and D5h Conformers Using IR Spectroscopy. J. Organomet. Chem. 2012, 713, 51-59.

(24) Coriani, S.; Haaland, A.; Helgaker, T.; Jørgensen, P. The Equilibrium Structure of Ferrocene. ChemPhysChem 2006, 7, 245-249.

(25) Dunitz, J. D.; Orgel, L. E.; Rich, A. The Crystal Structure of Ferrocene. Acta Crystallogr. 1956, 9, 373-375.

(26) Horcas, I.; Fernández, R.; Gómez-Rodríguez, J. M.; Colchero, J.; Gómez-Herrero, J.; Baro, A. M. WSXM: a Software for Scanning Probe Microscopy and a Tool for Nanotechnology. Rev. Sci. Instrum. 2007, 78, 013705.

(27) Kresse, G.; Furthmüller, J. Efficiency of Ab-Initio Total Energy Calculations for Metals and Semiconductors Using a Plane-Wave Basis Set. Comput. Mater. Sci. 1996, 6, 1550.

(28) Blöchl, P. E. Projector Augmented-Wave Method. Phys. Rev. B 1994, 50, 17953-17979.

(29) Kresse, G.; Joubert, D. From Ultrasoft Pseudopotentials to the Projector AugmentedWave Method. Phys. Rev. B 1999, 59, 1758-1775. 
(30) Perdew, J. P.; Burke, K.; Ernzerhof, M. Generalized Gradient Approximation Made Simple. Phys. Rev. Lett. 1996, 77, 3865-3868.

(31) Grimme, S. Semiempirical GGA-Type Density Functional Constructed with a LongRange Dispersion Correction. J. Comput. Chem. 2006, 27, 1787-1799.

(32) Graziano, G.; Klimes, J.; Fernandez-Alonso, F.; Michaelides, A. Improved Description of Soft Layered Materials with Van der Waals Density Functional Theory. J. Phys. Condens. Matter. 2012, 24, 424216.

(33) Tang, W.; Sanville, E.; Henkelman, G. A Grid-Based Bader Analysis Algorithm Without Lattice Bias. J. Phys. Condens. Matter 2009, 21, 084204.

(34) Tersoff, J.; Hamann, D. R. Theory and Application for the Scanning Tunneling Microscope. Phys. Rev. Lett. 1983, 50, 1998-2001.

(35) Tersoff, J.; Hamann, D. R. Theory of the Scanning Tunneling Microscope. Phys. Rev. $B$ 1985, 31, 805-813.

(36) Bocquet, M.-L.; Lesnard, H.; Monturet, S.; Lorente, N. Computational Methods in Catalysis and Materials Science; Wiley-VCH: Weinheim, Germany, 2009. 


\title{
Assembly of Ferrocene Molecules on Metal Surfaces Revisited
}

\author{
Maider Ormaza, ${ }^{*, \dagger}$ Paula Abufager, ${ }^{\ddagger}, N^{-}$Nicolas Bachellier, ${ }^{\dagger}$ Roberto Robles, ${ }^{\ddagger}, \S$ \\ Martin Verot,|| Tangui Le Bahers," Marie-Laure Bocquet, || Nicolas Lorente, ${ }^{\ddagger}, \S$ \\ and Laurent Limot ${ }^{\dagger}$
}

Institut de Physique et Chimie des Matériaux de Strasbourg, Université de Strasbourg, UMR CNRS 7504, 67034 Strasbourg, France, ICN2 - Institut Català de Nanociència $i$ Nanotecnologia, Campus UAB, 08193 Bellaterra (Barcelona), Spain, Instituto de Física de Rosario, Consejo Nacional de Investigaciones Científicas y Técnicas (CONICET) and Universidad Nacional de Rosario, Av. Pellegrini 250 (2000) Rosario, Argentina, CSIC -

Consejo Superior de Investigaciones Cientificas, ICN2 Building, Campus UAB, 08193 Bellaterra (Barcelona), Spain, and Université de Lyon, Université Claude Bernard Lyon 1, Centre National de Recherche Scientifique, ENS Lyon, 46 allèe d'Italie, 69007 Lyon Cedex 0\%, France

E-mail: maider.ormaza@ipcms.unistra.fr

\footnotetext{
${ }^{*}$ To whom correspondence should be addressed

†Institut de Physique et Chimie des Matériaux de Strasbourg, Université de Strasbourg, UMR CNRS 7504, 67034 Strasbourg, France

${ }^{\ddagger}$ ICN2 - Institut Català de Nanociència i Nanotecnologia, Campus UAB, 08193 Bellaterra (Barcelona), Spain

"Instituto de Física de Rosario, Consejo Nacional de Investigaciones Científicas y Técnicas (CONICET) and Universidad Nacional de Rosario, Av. Pellegrini 250 (2000) Rosario, Argentina

${ }^{\S}$ CSIC - Consejo Superior de Investigaciones Cientificas, ICN2 Building, Campus UAB, 08193 Bellaterra (Barcelona), Spain

"Université de Lyon, Université Claude Bernard Lyon 1, Centre National de Recherche Scientifique, ENS Lyon, 46 allèe d'Italie, 69007 Lyon Cedex 07, France
} 


\section{Supporting Information}

Figure 1 shows STM images acquired after deposition of ferrocene on $\mathrm{Cu}(100)$. In the large scale image of Figure 1(a) we see how for a ferrocene coverage below the monolayer, $\mathrm{Cu}(100)$ areas remain clean in between long-range ordered molecular layers. In Figure 1(b-d) we can distinguish the presence of the same compact and zigzag assemblies as on $\mathrm{Cu}(111)$. Table 1 presents the unit cell parameters for both arrangements as well as the apparent height of the molecular layer, which are almost identical to the parameters obtained for $\mathrm{Cu}(111)$. The fact that the substrate orientation does not influence the adsorption configuration of ferrocene suggests that the molecules have a weak coupling with the substrate, meaning that they are physisorbed. The low associative desorption temperature observed for this system, around $200 \mathrm{~K}$, also supports the idea of a feeble substrate-molecule coupling. Reinforcing this idea we find that the rotational domains observed for each configuration in the $\mathrm{Cu}(111)$ surface, $\left(0^{\circ}, 30^{\circ}, 60^{\circ}\right)$ for the compact and $\left(17^{\circ}, 50^{\circ}\right)$ for the zigzag, are also observed in $\mathrm{Cu}(100)$. This implies that the rotational domains are only related to the symmetry of the molecular layer itself and not to the one of the substrates.

Table 1: Apparent height of the molecular layer with respect to $\mathrm{Cu}(100)$ $\left(\mathbf{h}_{C u-F e C p_{2}}\right)$ and unit cell parameters $\left(\mathbf{a}_{1}^{\prime}, \mathbf{a}_{2}^{\prime}, \mathbf{b}_{1}^{\prime}, \mathbf{b}_{2}^{\prime}, \theta\right)$ of both compact and zigzag arrangements.

\begin{tabular}{ccccc} 
& $\mathbf{h}_{C u-F e C p_{2}}(\AA)$ & $\mathbf{a}_{1}^{\prime}(\AA)$ & $\mathbf{a}_{2}^{\prime}(\AA)$ & $\theta\left(^{\circ}\right)$ \\
\hline Compact & $3.1 \pm 0.1$ & $9.0 \pm 0.3$ & $16.0 \pm 0.3$ & $90 \pm 3$ \\
\hline \hline & $\mathbf{h}_{C u-F e C p_{2}}(\AA)$ & $\mathbf{b}_{1}^{\prime}(\AA)$ & $\mathbf{b}_{2}^{\prime}(\AA)$ & $\theta\left(^{\circ}\right)$ \\
\hline Zigzag & $3.1 \pm 0.1$ & $23.0 \pm 0.4$ & $13.0 \pm 0.4$ & $72 \pm 3$ \\
\hline
\end{tabular}



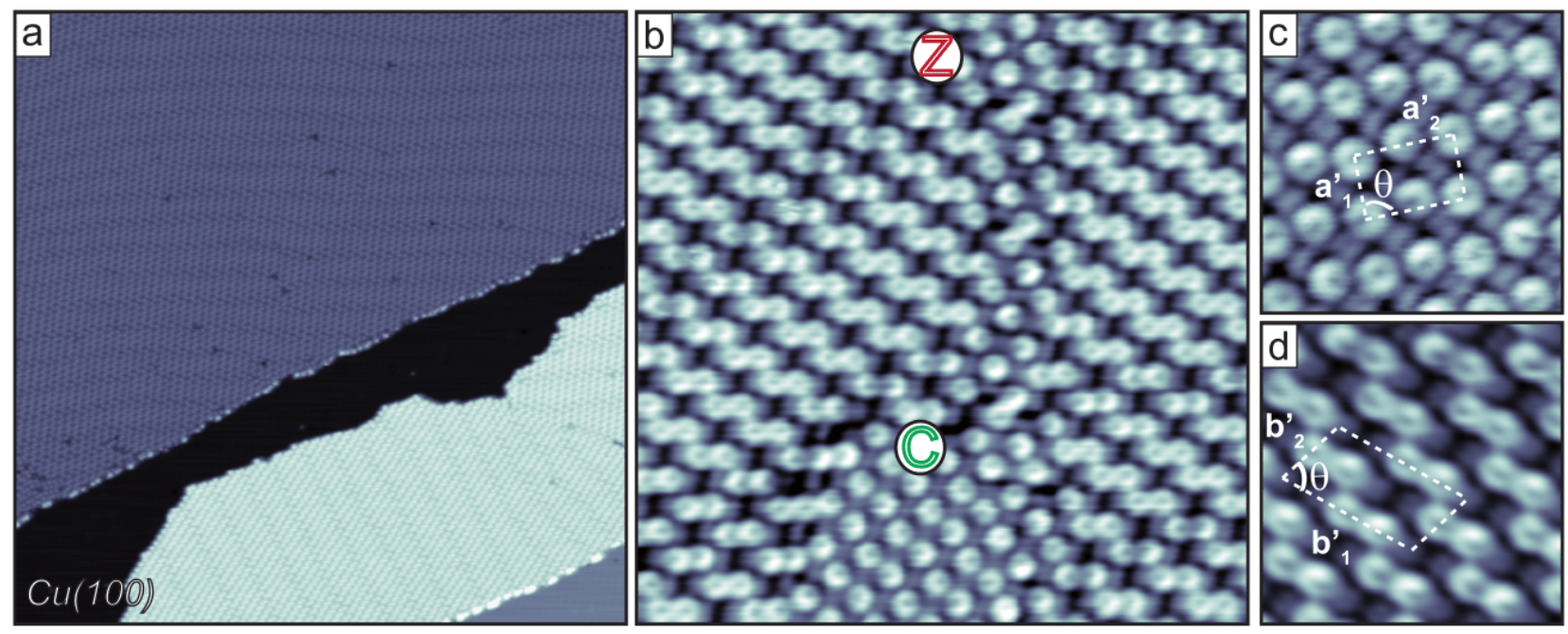

Figure 1: Ferrocene adsorption on $\mathrm{Cu}(100)$ : (a) Bare $\mathrm{Cu}(100)$ areas are observed in between well ordered ferrocene monolayers, (b) Both compact (C) and zigzag (Z) arrangements appear on the surface, (c-d) A zoom and the unit cells marked in white of both configurations, compact and zigzag respectively, are shown. Image parameters: (a) $(-1 \mathrm{~V}, 0.2 \mathrm{nA}),(\mathrm{b})(0.5 \mathrm{~V}$, $1 \mathrm{nA}),(\mathrm{c})(-1 \mathrm{~V}, 1 \mathrm{nA}),(\mathrm{d})(0.15 \mathrm{~V}, 1 \mathrm{nA})$. Image sizes: (a) $100 \times 100 \mathrm{~nm}^{2}$, (b) $15 \times 15 \mathrm{~nm}^{2}$, (c-d) $5 \times 5 \mathrm{~nm}^{2}$. 